\title{
Monitoring High Voltage Power Lines Using Efficient WSN
}

\author{
Khaled Al-Maitah, Batool Al-Khriesat, Abdullah Al-Odienat* \\ Department of Electrical Engineering, Faculty of Engineering, Mutah University, Jordan \\ Received March 13, 2020; Revised April 22, 2020; Accepted April 27, 2020
}

Copyright $\bigcirc 2020$ by authors, all rights reserved. Authors agree that this article remains permanently open access under the terms of the Creative Commons Attribution License 4.0 International License

\begin{abstract}
The power transmission line is one of most critical components in electrical power system. In order to enhance the reliability of the large power systems, an advanced and efficient Wireless Sensor Network (WSN) must be employed along power transmission line. However, time delay has emerged as one of the most critical issues in the design of WSN-based monitoring network of transmission lines. This paper proposes a new model for WSN for monitoring High Voltage Transmission Line (HVTL) which improves the needed time delay to transmit the measured quantity from transmission line towers to the control center. The proposed design considers the broadband power line communication (BPLC) technologies to transmit the collected data from group of towers to the nearest substation. To evaluate the performance of proposed model, the maximum time delay in data flow for the proposed model is determined in mathematical form and compared with other common models. The results of this paper demonstrate that the maximum time delay of proposed model less than the other common models, especially when advanced wireless communication, such as $4 \mathrm{G}$ and $5 \mathrm{G}$, is used between the relay nodes. Based on the simulation results, the proposed WSN-based transmission line monitoring is efficient, powerful, reliable, and applicable to meet the smart grid requirements.
\end{abstract}

Keywords Wireless Sensor Network (WSN), Broadband Power Line Communication, WSN Time Delay, HV Transmission Lines

\section{Introduction}

The electrical power system consists of hundred or even thousands of substations which are connected with power plants, lines, transformers and consumers. These substations are distributed across wide geographical areas
[1]. Moreover, power systems are now highly interconnected which allows the transfer of a large electrical energy over long distances far from the source of generation to locations suffer of lack in electrical energy [2] [3].

In power systems, transmission lines are critical components from the reliability and stability view point. There is a real need for an efficient monitoring system of the transmission lines to enhance the normal operation of the power system and avoid the major disconnections and blackouts [4]. in [5], a proposed WSN installed along the high transmission lines to monitor important parameters such as temperature and sag measurement. A advanced sensor networks may be used to monitor underground cables to reduce the maintenance costs and provide more accurate status information about the underground cables [6]. These sensors are connected to together through a wireless communication system to forms wireless sensor network WSN [7].

The WSN is a promising technology that provides the necessary and accurate data that would not have been possible with a single sensor. Since the WSN depends on the wireless communication technology; some important factors should be taken into account in the design of these networks such as time delay, reliability, and energy efficiency [8].

The concept of using wireless sensor in substation automation is proposed in [9], the authors used wireless sensors on the incoming and outgoing feeders in the substation to measure the current and to detect and locate the fault in the distribution network. The authors assumed in their work that the sensors are installed very close to the substations without communication between them.

The authors in [10] [11] were the first to propose wireless sensor technology in the power transmission line monitoring; they suggested the use of wireless sensors to estimate the line status and the conductor temperature based on intelligent technique. In their work, they assumed that the sensors are installed along the transmission line. 
However, the concept of the network did not appear in their work and communication between the sensors is not addressed. On the other hand, the authors in [12] [13] [14] [15] proposed a communication network structure between wireless sensors. they assumed that each tower in transmission line has its own relay node, each relay node have two communication modules, one of them for short range communication to collect the data from sensors of the tower and the other is a long range communication to send the collected data to neighboring relay node in the direction toward the substation. They assumed that the substations have a direct communication link with the main control center through fiber optic cables. It means that the relay nodes form a linear network between two substations located at the ends of transmission line.

The authors in [16] have shown that this communication model have a large time delay in data transfer. Therefore, they suggested that some of the relay nodes must have a direct wireless communication to the control center to reduce time delay and increase the data delivery speed in compatible with smart grid requirements.

The authors in ref. [17] identified the optimum placement of the direct link in relay nodes to minimize the time delay by dividing the relay nodes in transmission line into a number of groups, each group has one relay node connected to the control center through direct wireless link in order to reduce the time delay in data delivery, and evaluate the energy used in this link. The authors utilized a quadratic equation to find out the number of relay nodes which have a direct wireless link and subsequently the location of such nodes.

In ref. [18], further analysis is conducted on the optimal number and location of cellular modules based on weighted objective function to normalized network cost and transmission delay.

The authors in [19] proposed a combination scheme between WSN and radio frequency identification (RFID). They proposed fourth layer scheme between the relay node and control center.

From the literature review, it clear that the time delay is the one of the critical performance indicators in the design of WSN-based transmission line monitoring network. In our paper we propose a new model for WSN for HV transmission line monitoring enhanced with broadband power line communication BPLC technology. The maximum time delay for WSN models-based power transmission line monitoring is determined to validate the proposed model as compared to other available techniques in the literature.

The organization of the rest of this paper is as follows, in section II, WSN has been described in details. In section III, the proposed broadband network model (BPNM) is presented in details. In section IV, results and discussion, finally, the conclusions are presented in Section V.

\section{WSN Models}

Based on the information collected, smart grid (SG) could be a network that can easily accommodate self-healing, real time monitoring system, and fault tolerance with variations in consumption. WSNs have recently gained great interest in power system monitoring and automation.

Network models assist to adjust the wireless and wire line communications infrastructure of the power transmission line so that WSN data are timely delivered to the control center. Based on the established mathematical modeling in [17] [16] [20], there are two main WSN models; linear network model (LNM) and direct link network model (DLNM) as shown in figure 1 and figure 2, respectively.

\subsection{Linear Network Model (LNM)}

In Figure 1, substations 1 and 2 are connected to the control center via wire line connections, such as optical fiber. Depending on the length $(L)$ of the overhead HV network, the number of relay nodes $\left(n_{\text {relay }}\right)$ installed on the overhead HV transmission line will be $\left\lceil L / L_{\text {relay }}\right\rceil-1$, where $L_{\text {relay }}$ is the spacing between overhead towers along the transmission line. 


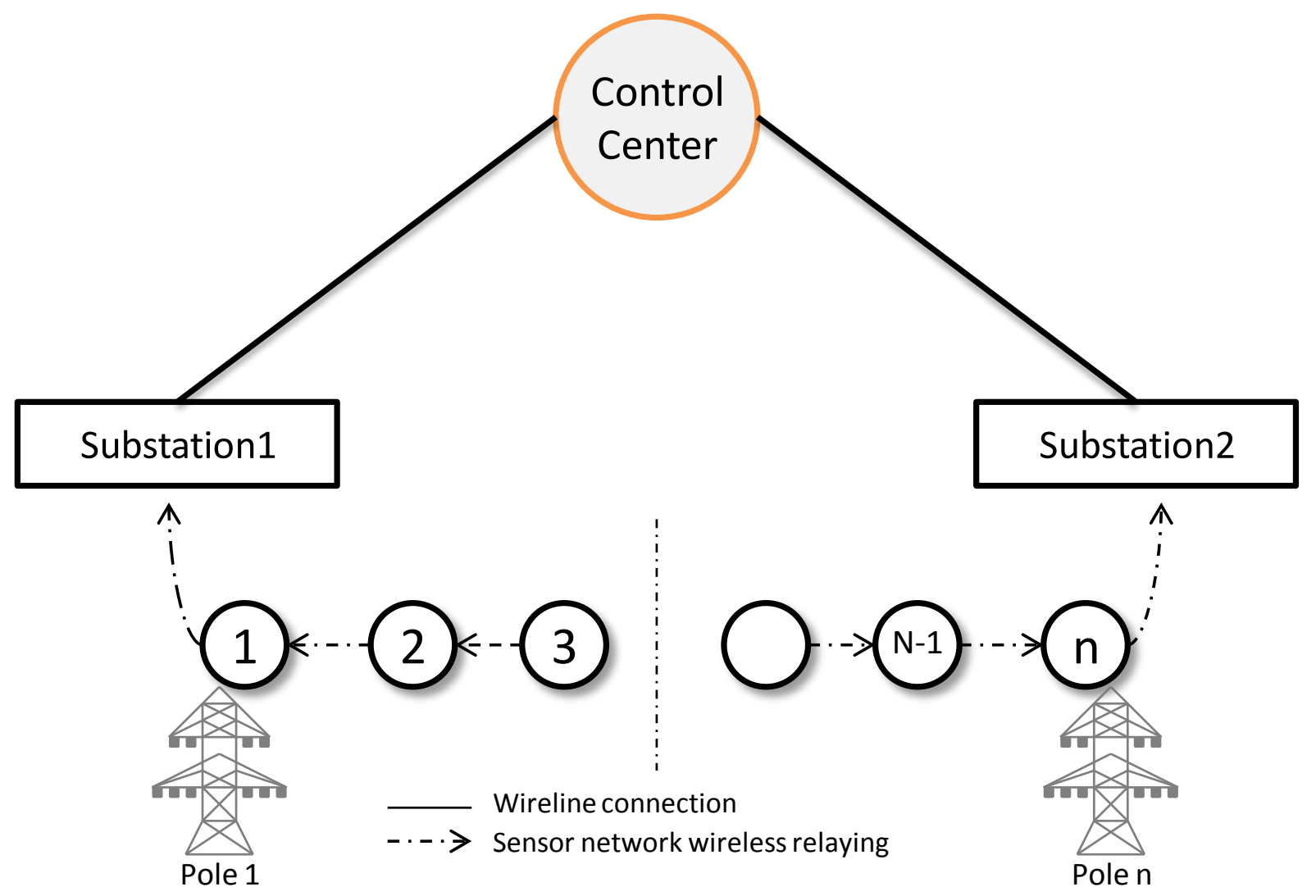

Figure 1. Linear network model LNM of WSN

In short-range communication, such as Bluetooth and ZigBee, the relay node receives the collected information from the surrounding sensors. The relay node then transmits the collected information wirelessly to the nearest relay node, which is relatively close to the substations at the ends of the overhead $\mathrm{HV}$ transmission line. According to $[17,8,3]$, the maximum time delay, which is the total time required for the data transmitted from the relay node $\left[n_{\text {relay }} / 2\right\rceil$ to arrive the nearest substation, is then given by,

$$
\mathrm{T}_{\mathrm{LNM}}=\frac{\mathrm{s}_{\mathrm{d}}}{\mathrm{R}_{\mathrm{i}}} \cdot\left[1+2+\cdots \frac{\mathrm{n}_{\text {relay }}}{2}\right]=\frac{\mathrm{s}_{\mathrm{d}}}{\mathrm{R}_{\mathrm{i}}} \cdot \sum_{\mathrm{x}=1}^{\mathrm{n}_{\text {relay }} / 2} \mathrm{x}
$$

Where $R_{\mathrm{i}}$ is the data rate in $\mathrm{KB} / \mathrm{sec}, S_{d}$ is the message size for each relay node, and $\mathrm{n}_{\text {relay }}$ is the total number of relay nodes. The summation in (1) can be simplified mathematically as,

$$
\sum_{\mathrm{x}=1}^{\mathrm{n}_{\text {relay }} / 2} \mathrm{x}=\frac{\left[\frac{\mathrm{n}_{\text {relay }}}{2}\right]\left[\frac{\mathrm{n}_{\text {relay }}}{2}+1\right]}{2}
$$

Then (1) can be written as,

$$
\mathrm{T}_{\mathrm{LNM}}=\frac{\left[\frac{\mathrm{n}_{\text {relay }}}{2}\right]\left[\frac{\mathrm{n}_{\text {relay }}}{2}+1\right] \cdot S_{\mathrm{d}}}{2 \cdot \mathrm{R}_{\mathrm{i}}}
$$

Where $R_{\mathrm{i}}$ is the data rate in $\mathrm{KB} / \mathrm{sec}, S_{d}$ is the message size for each relay node, and $\mathrm{n}_{\text {relay }}$ is the total number of relay nodes.

\subsection{Direct Link Network Model (DLNM)}

A more efficient WSN model, presented in [17], is based on the direct wireless links between the relay nodes and the control center. Since base stations can be several hundred kilometers away from the relay nodes, the long-range communication may use cellular technologies, such as GSM, 3G, 4G and 5G. According to this network model, shown in figure 2, r1 and r2 are the representative relay nodes and equipped with long relay node wireless communication capabilities. The two substations are considered representative nodes because they are always connected to the control center by a wire line communication such as fiber optic cables.

According to [17], to evaluate the maximum delay time, two other delay times should be determined in advance; the time delay of the relay node group $\left(\mathrm{T}_{\mathrm{rl}}\right)$, which is the time delay to collect the information in relay node $\mathrm{r} 1$ from the surrounding relay nodes located in the same group, and the time delay of the node group of substation $1\left(\mathrm{~T}_{\mathrm{si}}\right)$, which is the time delay to collect the information in substation 1 from the surrounding relay nodes and located in the same group.

Since all relay node groups have the same representative relay node wireless communication capabilities and the same symmetric frame with equal number of relay nodes, these relay node groups contribute with time delay $\left(T_{r 1}=\right.$ 
$\left.\mathrm{T}_{\mathrm{r} 2}\right)$. The time delay of the node group of substation $1\left(\mathrm{~T}_{\mathrm{s} 1}\right)$ is equal to the time delay of the node group of substation 2 $\left(\mathrm{T}_{\mathrm{s} 2}\right)$. Time delay of the relay node group $\left(\mathrm{T}_{\mathrm{r} 1}\right)$ is given by,

$$
T_{r 1}=\frac{K \cdot(K+1) \cdot S d}{2 \cdot R_{\text {Short }}}+\frac{K \cdot S d}{R_{\text {Short }}}+\frac{(2 . K+1) \cdot S d}{R_{\text {Long }}}
$$

Where $R_{\text {short }}$ is the short-range sensor network for the wireless relaying data rate in $\mathrm{KB} / \mathrm{sec}, S_{d}$ is the message size for each relay node, $R_{\text {long }}$ is the data rate of the long relay node wireless communication (direct link wireless communication) and $\mathrm{k}$ is the number of relay nodes in each group. Note that the middle term in (4) represents the time delay that one side of the relay node group should wait so that no collision occurs in the representative relay node due to the simultaneous transmission from the other side, the last term in (4) represents the time delay of sending all the collected data from the representative relay node to the control center through the long relay node wireless communication.

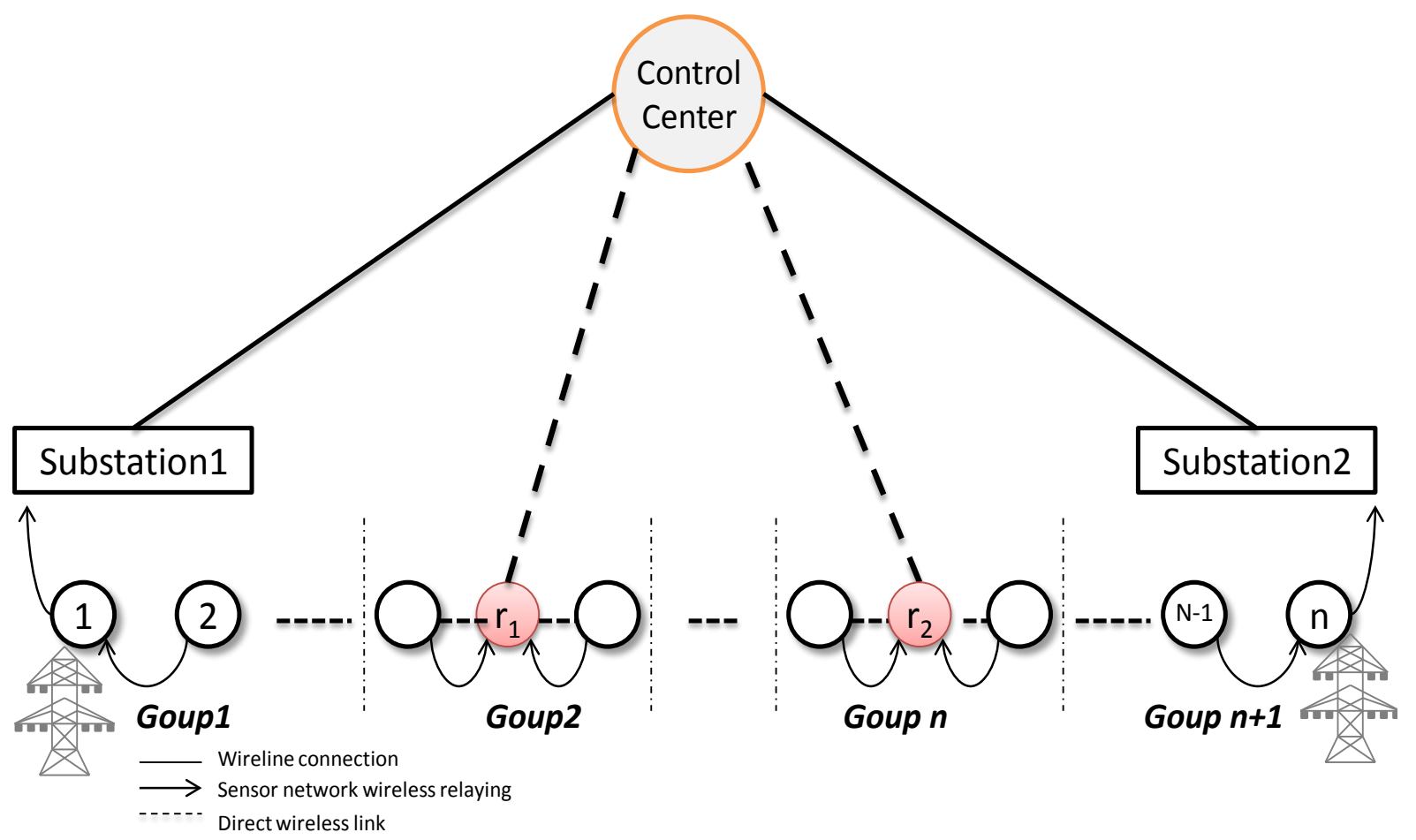

Figure 2. Direct network model DLNM of WSN 
To evaluate the time delay of the node group of substation $1\left(\mathrm{~T}_{\mathrm{s} 1}\right)$, firstly, the number of relay nodes connected with substation $1 \mathrm{~K}_{\text {sub } 1}$ must be determine by (5), where $\mathrm{n}_{\text {group }}$ is the number of groups, $\mathrm{k}_{\mathrm{g}}$ is the number of relay node in each group. Then the time delay to collect the information in substation 1 from the surrounding relay node $T_{s 1}$ is determined by (6)

$$
\begin{aligned}
K_{\text {sub } 1} & =\frac{n_{\text {relay }}-n_{\text {group }} \cdot\left(2 k_{g}+1\right)}{2} \\
T_{s 1} & =\frac{\left[K_{\text {sub } 1}\right]\left[\mathrm{K}_{\text {sub } 1}+1\right] \cdot S_{\mathrm{d}}}{2 \cdot \mathrm{R}_{\text {Short }}}
\end{aligned}
$$

Where $K_{\text {sub1 }}$ is the number of nodes connected with substation $1, R_{\text {Short }}$ is the data rate of the sensor network for wireless relaying and $S_{d}$ is the message size per relay node [24].

Using (4) and (6) the maximum delay time is easily determined by taking the maximum of these two times as in (7)

$$
\operatorname{Tmax}_{D L N M}=\operatorname{MAX}\left(T_{r 1}, T_{S 1}\right)
$$

where $\operatorname{MAX}\left\{T_{r 1}, T_{s 1}\right\}$ returns the highest value between $T_{r 1}$ and $T_{s 1}$.

\section{The Proposed Network Models}

Monitoring of overhead transmission lines is an important research area. WSN model used for that must be efficient and prepared to meet all current and future requirements of the modern smart grid. All available WSN models for transmission line monitoring such as LNM and DLNM suffer from the high time delay and high installation cost.

The conversion of the existing overhead $\mathrm{HV}$ transmission line to a smart overhead $\mathrm{HV}$ transmission line can be accomplished by the installation of BPLC broadband communication between the existing power lines [21].

BPLC network uses the existing line infrastructure of the transmission line for data communication. The main device in the BPLC network is the BPL units. Depending on the BPL network design and the network performance requirements, BPL units can be placed along the transmission line with spacing distances $L_{\mathrm{BPL}}$ varying from $1 \mathrm{~km}$ to $100 \mathrm{~km}$.

The BPL signal is injected into the transmission lines by the BPL modems of the units. BPL unit can be operated as a repeater to extract, regenerate, and inject the BPL signal, or as an aggregator to collect the data generated by the repeaters [22, 23]. The signals are usually injected into the over overhead transmission lines by [23]:

1. Coupling schemes for between two conductors.

2. Coupling schemes for one conductor ton ground.

The proposed network model is shown in figure 3 . The overhead $\mathrm{HV}$ lines spans a distances $L$ varies from $10 \mathrm{~km}$ to $100 \mathrm{~km}$, the BPL units can be placed at distances $L_{\mathrm{BPL}}$ ranging from $1 \mathrm{~km}$ to $50 \mathrm{~km}$. Depending on the previous distances, BPL units are installed across the overhead HV network $n_{\mathrm{BPL}}$, where $n_{\mathrm{BPL}}=\left\lceil L / L_{\mathrm{BPL}}\right\rceil-1$. The data are collected in the representative relay node, and then sent to the BPL unit to inject the signal into transmission line which will be received by the nearest substation. There is no direct connection between the representative nodes and the control center [24].

To evaluate the maximum time delay, two time delays should firstly be determined; the time delay of the relay node group by BPL units $\left(\mathrm{T}_{\mathrm{BPL} 1}\right)$ and the time delay of the node group of substation $1\left(\mathrm{~T}_{\mathrm{s} 1}\right)$. 


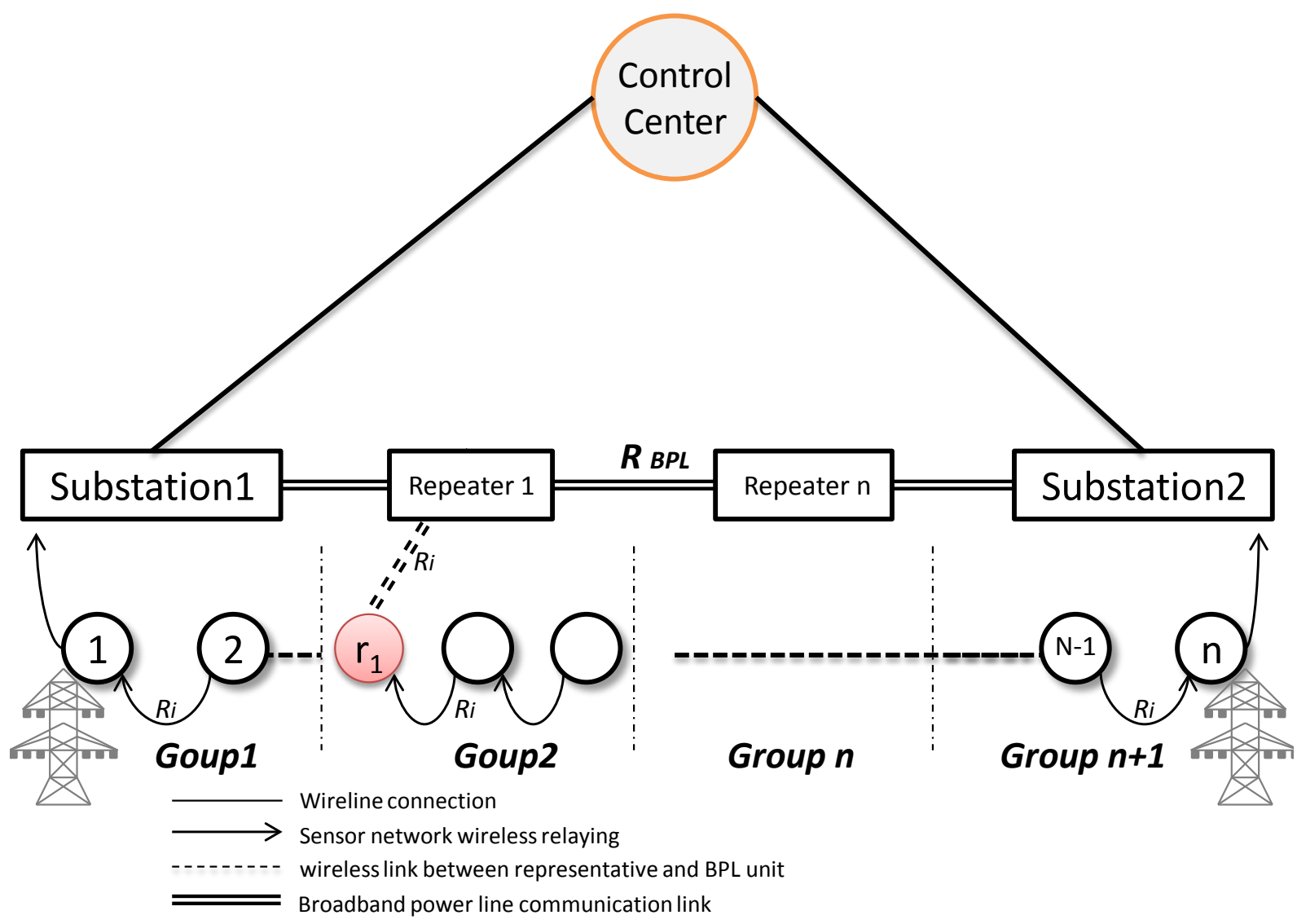

Figure 3. The Proposed network model of WSN (BPLNM)

Since all relay node groups have the same representative relay nodes, same wireless communication capabilities between either them or the BPL units, and same symmetric frame with equal number of relay nodes, these relay node groups will have the same time delay, the time delay of the relay node group $\left(\mathrm{T}_{\mathrm{BPL} 1}\right)$ is determined by (8)

$$
T_{B P L 1}=\frac{K \cdot(K+1) \cdot S d}{2 \cdot R_{\text {short }}}+\frac{(K+1) \cdot S d}{R_{\text {short }}}+\frac{\left.\left[n_{B P L} / 2\right] \cdot\left[\frac{\left[n_{B P L}\right.}{2}+1\right)\right] \cdot[K+1] \cdot S d}{R_{B P L}}
$$

Where $R_{\mathrm{BPL}}$ is the data rate of the BPL wire transmission line, $R_{\text {short }}$ is the short-range data rate of WSN relaying, and $S_{d}$ is the message size for each relay node. The time delay of the node group of substation $1\left(\mathrm{~T}_{\mathrm{s} 1}\right)$ is given by,

$$
T_{s 1}=\frac{\left[\mathrm{K}_{\text {sub1 } 1}\right]\left[\mathrm{K}_{\text {sub } 1}+1\right] . S d}{2 \cdot \mathrm{R}_{\text {Short }}}
$$

Where $K_{\text {sub1 }}$ is the number of nodes connected with substation1. The first term in (8) represent the total time delay to transmit the data from relay nodes to the representative node in group 1 located in the same group via short range communication technologies, the second term is the time delay to transfer the collected data from the representative node to the nearest BPL unit, whereas the last term is the overall time for the data of the BPL unit $i_{\text {repeater }}$ to reach the closest substation [24].

Based on (8) and (9), the maximum time delay for the proposed PBLNM is given by (10)

$$
\operatorname{Tmax}_{P B L N M}=\operatorname{MAX}\left\{T_{P B L 1}, T_{S 1}\right\}
$$

where $\operatorname{MAX}\left\{T_{P B L 1}, T_{S 1}\right\}$ returns the highest value between $T_{P B L 1}$ and $T_{S 1}$.

\section{Results and Discussion}

In order to validate the proposed model, a power transmission line with 1 to $100 \mathrm{~km}$ length has been modeled by MATLAB. The simulation results of various network model of WSNs for power transmission line monitoring has been done to evaluate the performance of LNM, DLNM and the proposed PBLNM model. To make a comparison between the proposed model and others, the maximum time delay has been determined for all models at different transmission line lengths with various wireless communication technology such as GSM, 3G, 4G and 5G.

\subsection{Linear Network Model (LNM) Performance}

LNM is simple, the calculation of the maximum time delay done by equation (3). In Figure 4, the maximum time delay of LNM is plotted versus the length of the transmission line for $23 \mathrm{~KB}$ message sizes per relay node. 


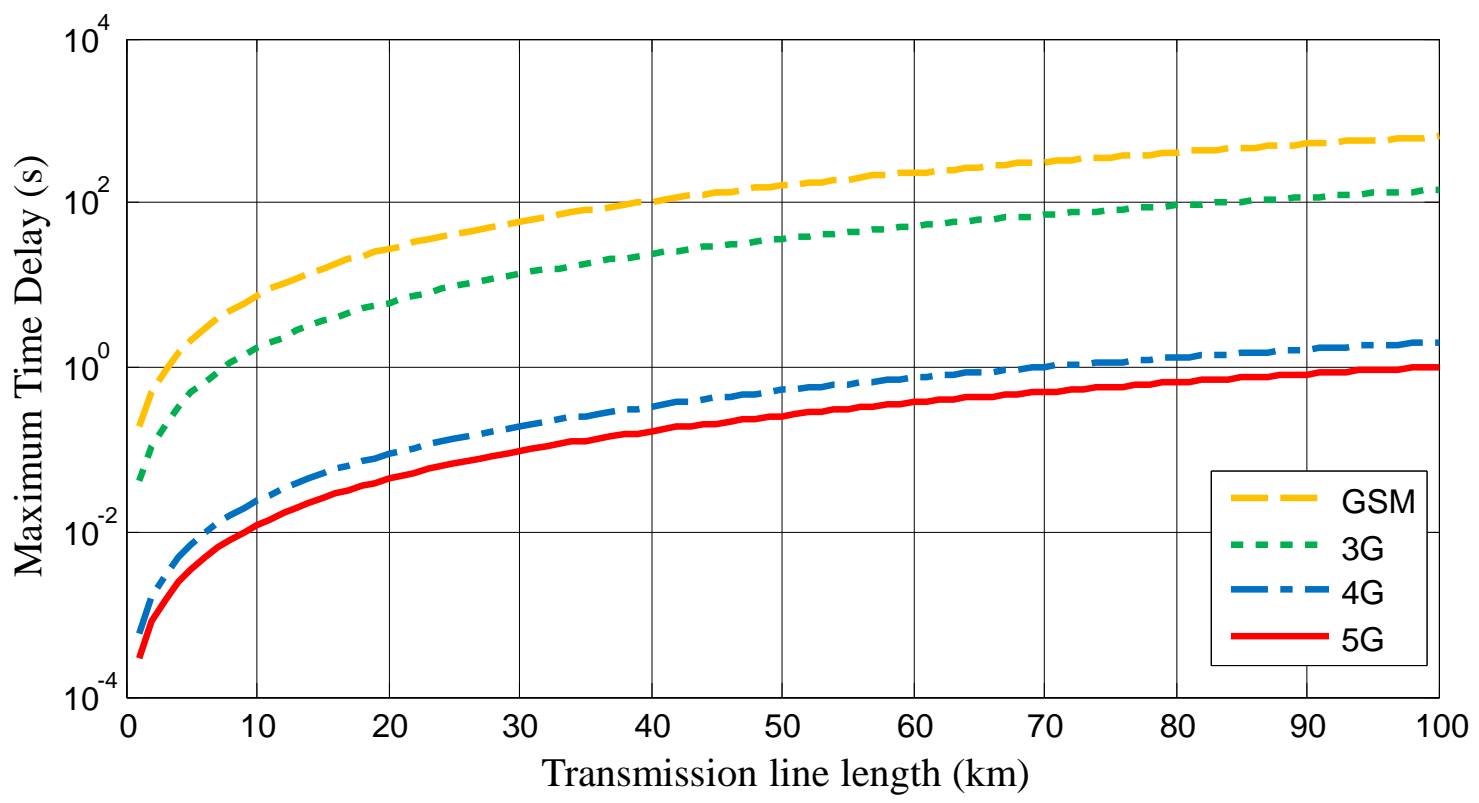

Figure 4. Maximum time delay in second for LNM with GSM, 3G, 4G, and 5G technologies using 32 KB data size for each relay node

The distance between relay nodes is assumed to be $1 \mathrm{~km}$, GSM, 3G, 4G and 5G as a wireless communication technology are used in the system test. The maximum time delay by using $5 \mathrm{G}$ is the lowest, it's about 1 second at 100 $\mathrm{km}$ transmission line length. LNM is characterized by high time delay specially when the transmission line length is high. The maximum time delay results indicate that LNM is suitable for monitoring short distance transmission lines with tolerance in latency. This of course does not meet the requirement of real time monitoring in smart grid applications.

\subsection{Direct Line Network Model (DLNM) Performance}

In DLNM, WSN requirements dictate the number of relay node groups and the number of the relay nodes in each group. By increasing the number of relay node groups, the maximum delay time is reduced.

In Figure 5, the maximum delay time of LNM is plotted versus the length of the overhead $\mathrm{HV}$ network for $32 \mathrm{~KB}$ message sizes per relay node when the distance between relay nodes is equal to $1 \mathrm{~km}$ using GSM, 3G, 4G and 5G as wireless technologies. The maximum time delay by using $5 G$ is the lowest one, it's about 0.22 second for $100 \mathrm{~km}$ transmission line length. The results show that DLNM is more efficient than LNM in term of time delay. Therefore, the DLNM is more applicable and suitable for future smart grid applications than LNM. 


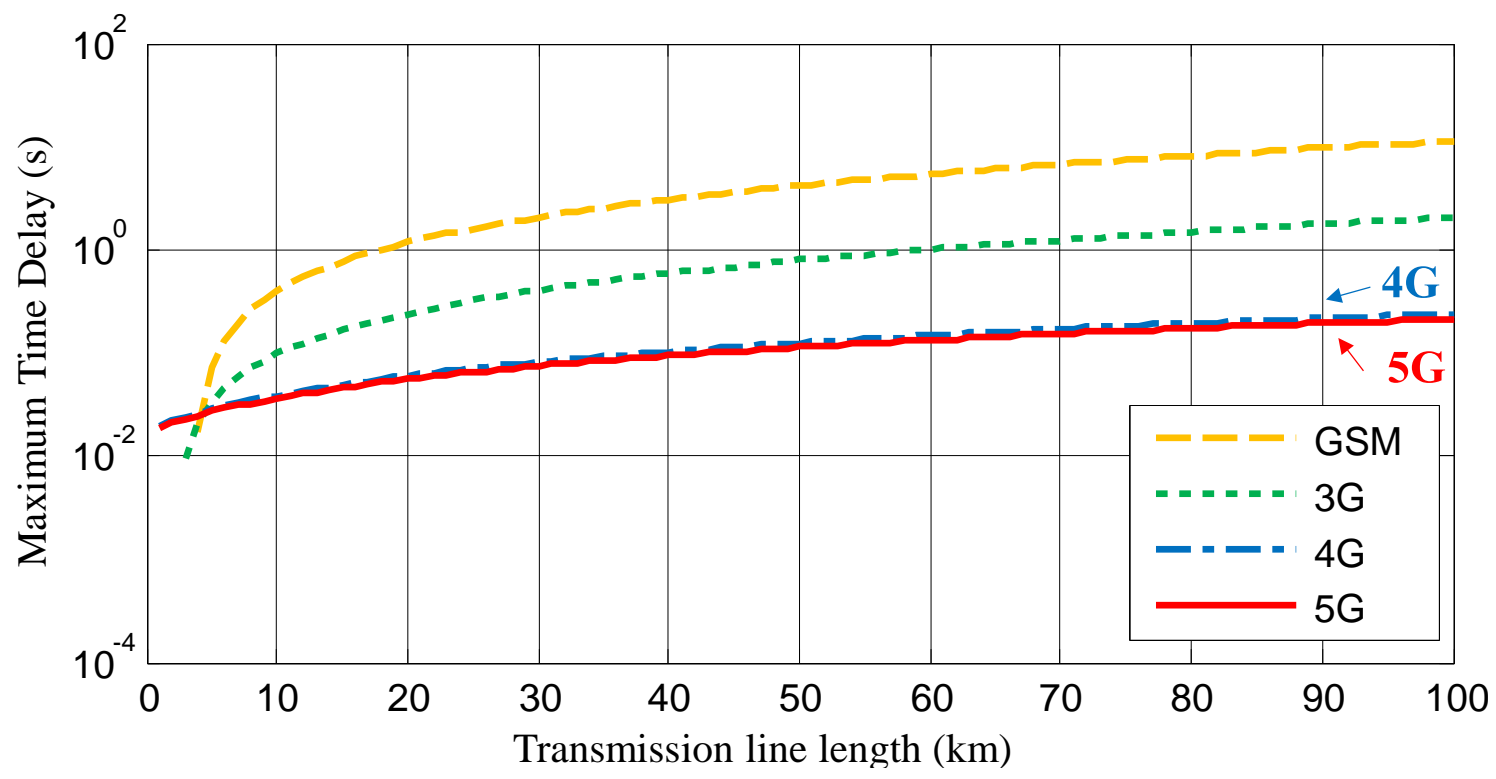

Figure 5. Maximum time delay in seconds for DLNM with GSM, 3G, 4G, and 5G technologies using 32 KB data size for each relay node

\subsection{Broadband Line Network Model (BPLNM) Performance}

According to the previous discussion in section III, and in order to validate the proposed model of transmission line monitoring, the maximum delay time of the proposed model (BPLNM) is plotted versus the length of the overhead $\mathrm{HV}$ network for $32 \mathrm{~KB}$ message sizes per relay node when the distance between relay nodes is equal to $1 \mathrm{~km}$ and using GSM, 3G, 4G and 5G as wireless technologies, the number of BPL units installed among the transmission line is assumed to be 10, where the spacing between BPL units is $1 \mathrm{Km}$. Figure 6 represent the maximum time delay of proposed model with different communication technologies.

The maximum time delay at $100 \mathrm{~km}$ transmission line is about $68 \mathrm{~ms}$ for 5G technology.

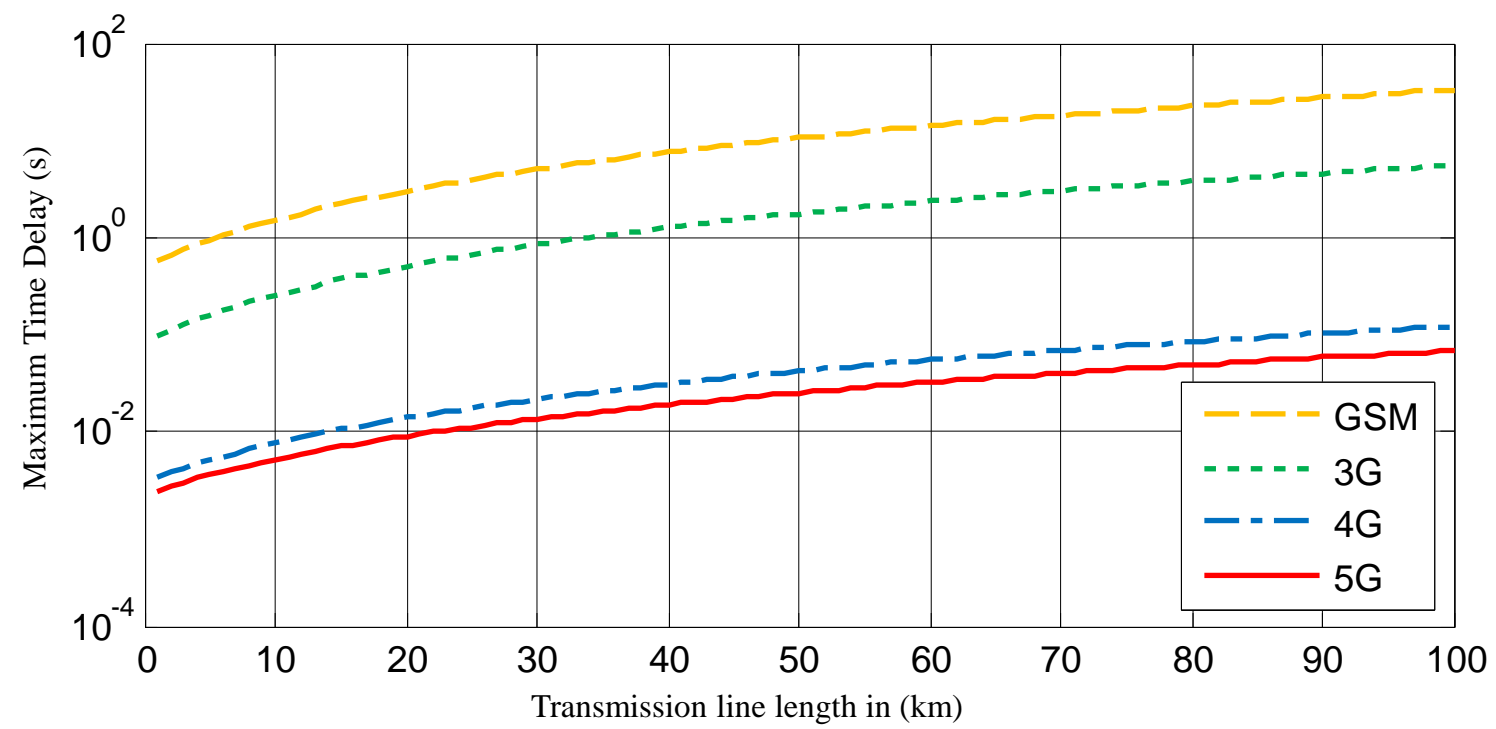

Figure 6. Maximum time delay in second for DLNM with GSM, 3G, 4G, and 5G technologies using $32 \mathrm{~KB}$ data size for each relay node

Generally, to make the comparison clearer, table 1 represent the maximum time delay at 50 and $100 \mathrm{~km}$ transmission line length for all three models with different communication technologies, which are GSM, 3G, 4G, and 5G. 
Table 1. Maximum Time Delay for Various Communication Technologies for All Models

\begin{tabular}{|c|c|c|c|c|c|c|c|c|}
\hline Com. & \multicolumn{2}{|c|}{ GSM } & \multicolumn{2}{c|}{ 3G } & \multicolumn{2}{c|}{ 4G } & \multicolumn{2}{c|}{$5 \mathrm{~F}$} \\
\hline T.L length (km) & 50 & 100 & 50 & 100 & 50 & 100 & 50 & 100 \\
\hline LNM (Sec) & 162 & 637 & 36 & 143 & 0.52 & 2.04 & 0.26 & 1.02 \\
\hline DLNM (Sec) & 4.1 & 11.27 & 0.7 & 2 & 0.12 & 0.22 & 0.11 & 0.22 \\
\hline BPLNM (Sec) & 10 & 33 & 1.7 & 5.5 & 0.04 & 0.12 & 0.025 & 0.06 \\
\hline
\end{tabular}

Firstly, at any transmission line length and with any communication technology the LNM is the worst model, that because it has the largest value of maximum time delay. For example, the maximum time delay of LNM when the transmission line is $100 \mathrm{~km}$ and $4 \mathrm{G}$ is used as a communication technology was about 2.04 second, which is large than the maximum time delay for DLNM and BPLNM which are 0.22 and 0.12 second, respectively.

Secondly, when the GSM or 3G communication technologies are used the DLNM has lower time delay. For example, the maximum time delay for DLNM and BPLNM at $100 \mathrm{~km}$ transmission line when $3 G$ technology is used was about 2 and 5,5 second, respectively. From the other hand, when the $4 \mathrm{G}$ and $5 \mathrm{G}$ are used as a communication technology the proposed model (BPLNM) has the lowest time delay. That is because the maximum delay time of BPLNM is mainly depending on the maximum delay time of the relay node group via BPL units.

Based on the above results the proposed model is the best one for transmission line monitoring and applicable for medium and long transmission line when it is supported with high communication technologies such as 4G and 5G.

\subsection{Comparisons between the Network Models}

Based on the above discussion, the maximum time delay for the proposed model (BPLNM) less than the maximum time delay in other network models (LNM and DLNM). In other hand, there are several considerations must to be taken in account in practical implementation of any WSN-based transmission line monitoring, such as cost, cyberattack, and energy consumption. In this section, these considerations have discussed and compared between the WSN network models.

i. The cost in WSN network models can be can be divided to the installation and operation cost. The installation cost mainly involves the equipment cost. In other hand the operation cost involves the maintenance and energy consumption cost. Although, the LNM has less installation cost, but the operation cost is largest due to the high energy is need in hop by hop transmission form. In DLNM the installation cost larger than the LNM due the needed to upgrade some of relay node to the representative node. Further on, in DLNM long wireless communication is needed, which is increase the energy consumption and therefor it effects on the operation cost. In the proposed model (BPLNM) there is no need to long wireless communication, therefor, there is no additional operational cost. Using the existence infrastructure of overhead transmission line reduces the installation.

ii. The using of power line in the proposed model offer more data security from the cyberattack due to the fact the physical access in power line is not easy. Although, the using of power line in proposed model increase from the system resistance to the attack, but additionally software are needed to reach a high level of security.

iii. Since the sensor node has limited energy resources, a key issue in WSN is the energy required to an efficient operation for long time duration. Where, it is not practical to change the battery in continuous manner. In DLNM there are high energy consumption due to the need of long wireless connection, Contrary to the proposed model (BPLNM) which design to get rid of long wireless communication therefor enhances from decrease the needed energy in the relay node.

\section{Conclusions}

Since the WSN Wireless Sensor Network (WSN) is a technology that allows sensors to coordinate among each other, the time delay is the critical parameter to evaluate the performance of WSN-based transmission line monitoring network. This paper presents a new WSN model, named BPLNM, based on broadband power line communication. The proposed model BPLNM has been compared with two other network models from the relevant literature (i.e., LNM and DLNM). As a result of this paper, the BPLNM is the suitable network model when advanced communication technology is used for power transmission line monitoring. The proposed network model is more applicable to meet the smart grid requirements than LNM and DLNM.

Since the energy consumption is an important issue in any WSN model, this work can be extended to decrease the energy absorbed by communication module especial when the representative node is chosen in optimal location in the proposed model. 


\section{REFERENCES}

[1] J. B. Anderson, Electrical Power Systems, New York: The Institute of Electrical and Electronics Engineers, 1983.

[2] M. Eremia and M. Shahidehpour, Handbook of Electrical Power System Dynamics: Modeling, Stability, and Control, Canada: IEEE, 2013.

[3] Z. Zhang, L. Peng, H. Wang, J. Sun, T. Chang and L. Zhuo, A reliable routing method for transmission monitoring wireless sensor network, in IEEE Conference on Energy Internet and Energy System Integration (EI2), Beijing, 2017.

[4] Y. Cui, X. Song, L. Zhao, H. Yuan, G. Wu and C. Wang, WSN-Based Measurement of Ion-Current Density Under High-Voltage Direct Current Transmission Lines, IEEE Access , vol. 7, pp. 10947-10955, 2019.

[5] C. Bernhauer, H. Bohmer, S. Kornhuber, S. Markalous, M. Muhr and T. Strehl, Temperature measurement on overhead transmission lines (OHTL) utilizing surface acoustic wave (SAW) sensors, in International Conference on Electricity Distribution, Vienna, Austria, 2007.

[6] V. Sood, D. Fischer, J. Eklund and T. Brown, Developing a communication infrastructure for the smart grid, in IEEE Electrical Power and Energy Conference (EPEC 2009), Montreal, 2009.

[7] S. Malhara and V. Vittal, Mechanical state estimation of overhead transmission lines using tilt sensors, IEEE Trans. Power Syst., vol. 25, no. 3, pp. 1282-1290, vol. 25, no. 3, pp. 1282-1290, 2010.

[8] P. Kong, K.-S. Tseng, J.-A. Jiang and C.-W. Liu, Robust Wireless Sensor Networks for Transmission Line Monitoring in Taiwan, in IEEE International Conference on Communications, Control, and Computing Technologies for Smart Grids (SmartGridComm), Aalborg, 2018.

[9] Lehtonen and M. Nordman, A wireless sensor concept for managing electrical distribution networks, in IEEE Power Systems Conference, New York, 2004.

[10] Y. Yang, F. Lambert and a. D. Divan, Power line sensor net-A new concept for power grid monitoring, in IEEE Power Engineering Society General Meeting, Montreal, 2006.

[11] Y. Yang, F. Lambert and a. D. Divan, A survey on technologies for implementing sensor networks for power delivery systems, in IEEE Power Engineering Society General Meeting, Tampa, 2007.

[12] J. Chen, S. Kher and a. A. K. Somani, Energy efficient model for data gathering in structured multiclustered wireless sensor networks, in IEEE International Performace, Computing, and Communications Conference, Phoenix, AZ, 2006.

[13] V. Vittal and G. Manimaran, Application of sensor network of secure electric energy infrastructure, IEEE Transactions on Power Delivery, vol. 22, no. 2, pp. 1021-1028, 2007.

[14] P. Ramachandran, V. Vittal and G. T. Heydt, Mechanical state estimation for overhead transmission lines with level spans, IEEE Transactions on Power Systems, vol. 23, no. 3, pp. 908-915, 2008.

[15] Q. Huang, Y. Chen and a. C. Zhang, Design of an adaptive on-load deicing scheme for overhead power transmission line, in IEEE Power Engineering Society General Meeting, Calgary, 2009.

[16] K. Hung, W. Lee, V. Li, K. Lui, P. Pong, K. Wong, G. Yang and a. J. Zhong, On Wireless Sensors Communication for Overhead Transmission Line Monitoring in Power Delivery Systems, in 2010 First IEEE International Conference on Smart Grid Communications, Gaithersburg, 2010.

[17] Y. Wu, L. Cheung, K. Lui and a. P. W. T. Pong, Efficient Communication of Sensors Monitoring Overhead Transmission Lines, IEEE Transactions On Smart Grid, vol. 3, no. 3, pp. 1130-1136, 2012.

[18] M. Li, X.-B. Chi, X.-C. Jia and J.-L. Zhang, WSN-Based Efficient Monitoring for Overhead Transmission Line in Smart Grid, in 35th Chinese Control Conference, Chengdu, 2016.

[19] F. Deng, P. Zuo, K. Wen and X. Wu, Low Delay Technology Research of Transmission Line Tower Monitoring Network Integrating WSN and RFID, IEEE Access, vol. 7, pp. 111065-111073, 2019.

[20] G. Bag, R. Majumder and K.-H. Kim, Low Cost Wireless Sensor Network in Distributed Generation, in 2010 First IEEE International Conference on Smart Grid Communications, Gaithersburg, 2010.

[21] W. Zhu, X. Zhu, E. Lim and a. Y. Huang, State-of-art power line communications channel modelling, Procedia Computer Science, vol. 17, pp. 563-570, 2013.

[22] A. G. Lazaropoulos, Broadband transmission characteristics of overhead high-voltage power line communication channels, p. Progress in Electromagnetics Research, 2012.

[23] A. G. Lazaropoulos, Review and progress towards the common broadband management of high-voltage transmission grids: model expansion and comparative modal analysis, ISRN Electronics, 2012.

[24] A. G. Lazaropoulos, Wireless sensor network design for transmission line monitoring, metering, and controlling: Introducing broadband over power lines-enhanced network model (BPLeNM), International Scholarly Research Notices, vol. 2014, p. 22, 2014. 